\title{
Experimental Evidence of Spontaneous Transfer of Coherence in a Driven Degenerate Two-level System under the Action of a Linearly or a Circularly Polarized Probe
}

\author{
L. Spani Molella, K. Dahl, R.-H. Rinkleff, K. Danzmann \\ Albert Einstein Institute, Max Planck Institute for Gravitational Physics \\ and Institut für Gravitationsphysik, Leibniz Universität Hannover \\ Callinstraße 38, D-30167 Hannover, Germany. \\ Tel: +49 511762 4912; Fax: +49 511762 2784; E-mail: luca.spani.molella@aei.mpg.de
}

\begin{abstract}
Electromagnetically induced absorption (EIA) in a degenerate closed transition was measured under the action of a probe and a coupling laser of various polarizations giving rise to different possible $\mathrm{N}$-systems or multi-level systems. While the probe shows EIA the coupling is mainly characterized by "absorption within transparency". However, for certain lasers' intensity ranges, a switch between "absorption within transparency" and "transparency within transparency" in the coupling absorption spectra was measured for the first time.

(c) 2007 Optical Society of America

OCIS codes: (270.1670) Coherent optical effects; (300.1030) Absorption
\end{abstract}

Electromagnetically induced absorption (EIA) has recently been the object of a number of papers, both experimental and theoretical, mainly discussing two linearly polarized lasers of orthogonal polarization. However, the most simple configuration in which EIA arises is the so-called N-system [1]. In the approximation of a strong coupling laser, an easy way to experimentally realize such a system is to use a circularly polarized coupling laser and a linearly polarized probe laser, simultaneously driving a closed degenerate two-level system.

This configuration has been relatively neglected in experimental work. In particular, to our knowledge, no experimental measurements of the coupling laser exist in such an experimental configuration. Similarly absent are measurements of the coupling laser absorption level in case of circularly polarized probe and coupling laser of right-hand and left-hand polarisation.

In this contribution we present absorption spectra of the coupling and the probe laser in case of either circularlinear or circular-circular polarisation. The measurements were performed within the $\mathrm{D}_{2}$ line of caesium, on the hyperfine transition $6 \mathrm{~s}^{2} \mathrm{~S}_{1 / 2}, \mathrm{~F}=4$ to $6 \mathrm{p}^{2} \mathrm{P}_{3 / 2}, \mathrm{~F}=5$. To minimize the Doppler broadening of the signals the authors utilized an atomic beam of caesium atoms propagating perpendicularly to the direction of propagation of the lasers. The lasers were organized in a heterodyne interferometer. Further details on the setup can be found in $[2,3]$.

As for the case of double-linear orthogonal polarisations the coupling spectra are characterized by a broad transparency window mainly accompanied by a narrow feature at the two-photon resonance. In case of double linear polarisation we named this behaviour absorption within transparency [4,5], because the absorption of the coupling laser goes back to its starting level-i.e., the coupling laser absorption coefficient when the probe is far off-resonant - once the probe and the coupling frequencies match those required for the two-photon resonance. Similar spectra were measured either in the case of circularly polarized lasers of right-hand and left-hand polarisation, and in the linear-circular case. All these measurements confirm the interpretation of electromagnetically induced absorption as a phenomenon induced by spontaneous transfer of coherence, at least as long as the probe and the coupling laser do not share the same polarisation, as pointed out in [1].

The spectra were measured for various intensity of both lasers. Depending on the intensity ranges measured it was possible to evidence a broadening of the broad transparency signals accompanied by a variation in the intensity of the central narrow-line feature.

The work was made possible through the financial support of the grant No. SFB407 of the German Research Foundation (Deutsche Forschungsgemeinschaft).

[1] C. Goren, A. D. Wilson-Gordon, M. Rosenbluh, and H. Friedmann, “Atomic four-level N systems”, Phys. Rev. A 69, 053818 (2004)

[2] L. Spani Molella, R.-H. Rinkleff, and K. Danzmann, "Role of the coupling laser in electromagnetically induced transparency", Phys Rev. A 72, 041802(R) 1-4 (2005)

[3] L. Spani Molella, R.-H. Rinkleff, and K. Danzmann, "Spectral measurement of the cesium $\mathrm{D}_{2}$ line with a tunable heterodyne interferometer", Spectrochim. Acta A 63, 987-993 (2006)

[4] L. Spani Molella, G. Kuhn, R.-H. Rinkleff, K. Danzmann, "Electromagnetically induced transparency, electromagnetically induced absorption and giant Kerr effect in closed degenerate two-level systems", SPIE Proc. 6604, 66040B (2007)

[5] L. Spani Molella, "Nonlinear spectroscopy of closed degenerate two-level systems", PhD Thesis, Hannover 2006 